\title{
EFFECT OF PORE SIZE DISTRIBUTION AND TEMPERATURE ON THE CATALYST TORTUOSITY
}

\author{
Sepehr Sadighi ${ }^{*}$, Majid Bahmani ${ }^{2}$, Seyed Reza Seif Mohadecy ${ }^{2}$ \\ ${ }^{I}$ Reaction Engineering Department, Catalysis Research Center and Nano Technology, Research Institute of \\ Petroleum Industry (RIPI), Tehran, IRAN \\ ${ }^{2}$ Applied Chemistry Group, Chemistry Department, University of Tarbiat \\ Moalem, Tehran, IRAN
}

Received 5 January 2013; received in revised form 17 September 2013

\begin{abstract}
Effect of temperature gradients, for an exothermic reaction, inside a catalyst pellet and the pore size distribution on the value of tortuosity factor is studied. The structure of the catalyst pellet is simulated using a two dimensional pore network. Material and energy balances for the pore network are written and discretised using a finite difference scheme. Tortuosity factors obtained for the isothermal and non-isothermal pellets are compared. Significant variation of the estimated tortuosity factor with internal particle temperature gradient and pore size is obtained.
\end{abstract}

Keywords: Tortousity Factor; Pore Networks; Catalyst; Exothermic Reaction.

\section{INTRODUCTION}

The capillary model is the most common approach to describe diffusion and reaction phenomena occurring in the porous media. Thiele (1939) was the first to model catalyst pores as cylinders of the same diameter lying parallel to each other in a plane. Petersen (1957) improved the capillary model by considering the axes of the cylinders placed randomly in a plane. Improvements on these models of porous media included the interconnection of the pores in two dimensions by Fatt (1956). Beeckmn and Froment (1980) used a Bethe lattice representation of the porous medium in which each pore has a connectivity of three. Cylindrical pores of random diameters placed in two dimensions were used by Sharrat and Mann (1987) for investigation of deactivation of FCC catalysts. Hollewand and Gladden (1992) used randomly connected pores and Rieckman and keil
(1999) used regular three dimensional pore networks.

Due to the tortuous paths offered by the catalyst pores, diffusion of reactants is hindered and hence an effective diffusivity is used:

$$
D_{\text {eff }}=\frac{1}{\tau} \int_{0}^{\infty} D(r) f(r) d r=\frac{\bar{D}(r) \varepsilon}{\tau}
$$

Where $f(r) d r$ is the fraction of particles having a radius between $[\mathrm{r}, \mathrm{r}+\mathrm{dr}]$ and $\tau$ is the tortuosity factor.

Both dynamic and steady state methods are available for the measurement of effective diffusivity and it is more common to use the steady state method of the Wicke-Kallenbach. In this method the reactant is allowed to diffuse through the material under the no reaction condition. 
There is disagreement with regards to the values of tortuosity factor for catalyst supports for example Satterfield and Cadle (1968) reported values of 2.8 to 7.3 for a large number of commercial catalyst supports and also indicated that majority of catalyst supports have a tortuosity of 4 .

Lack of agreement on values for the tortuosity factor has been a matter of several theoretical studies. Otani and Smith (1966) were the first to question the applicability of using the tortuosity factors measured under diffusion only methods to the reactive condition. Sharratt and Mann (1987) used a two dimensional network of randomly sized pores and solved the diffusion reaction equation to conclude that the tortuosity factor was a function of the Thiele modulus i.e it is reaction dependent. Sahimi (1988) and Hollewand and Gladden (1991) also obtained the same conclusion using three dimensional pore network models.

All the previous works described assumed isothermal pellets and neglected the effect of internal variation of temperature in the catalyst and for highly endothermic or exothermic reactions temperature gradients in the catalyst lead to such phenomena as steady state multiplicities and therefore spatial variation of the reaction rate in the catalyst is expected. The previous workers have indicated the variation of tortuosity factor with the Thiele modulus and the main purpose of this work is to study the effect of internal temperature gradients in the catalyst pellet on the values of tortuosity factor.

\section{MODEL DEVELOPMENT}

In this work a two dimensional network of pores as shown in figure (1) has been developed which takes into account internal variation of temperature and concentration within the catalyst pores.
Simulation for isothermal and nonisothermal pellets have been carried out and compared.

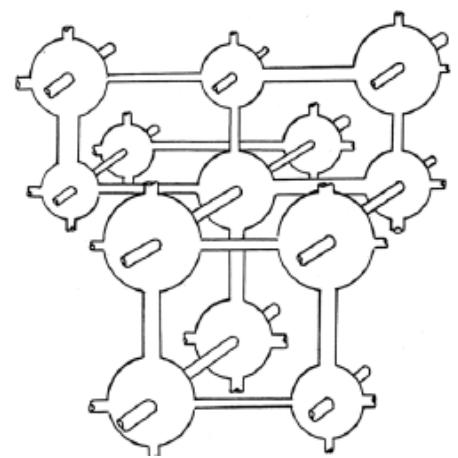

Figure 1: Two Dimensional Pore Network

The material and energy balance for a cylindrical pore is written as:

$\bar{D} \frac{d^{2} C}{d x^{2}}-\frac{2}{r_{p}} r(C, T)=0$
$\lambda_{e} \frac{d^{2} T}{d x^{2}}-\frac{2}{r_{p}} r(C, T) \Delta H=0$

The interior nodes are mixing places and no reaction takes place there and an equation similar to the Kirchoff's law is applied:

$$
\sum_{i=1}^{n} J_{i}=0
$$

At the outer nodes the material and heat fluxes from the bulk phase is given by:

$$
\begin{aligned}
& D \frac{d C_{s}}{d x}=k_{g}\left(C_{b}-C_{s}\right) \\
& \lambda \frac{d T_{s}}{d x}=h\left(T_{b}-T_{s}\right)
\end{aligned}
$$

The derivatives were discretised by means of finite difference formula. For the interior pores the central difference formula was used:

$\frac{d^{2} C}{d x^{2}}=\frac{C_{k+1}-2 C_{k}+C_{k-1}}{\Delta x^{2}}$ 
At the pore ends one-sided difference formula was used:

$$
\begin{aligned}
& \left.\frac{d C}{d x}\right|_{x=0}=\frac{-3 C_{o}+4 C_{1}-C_{2}}{2 \Delta x} \\
& \left.\frac{d C}{d x}\right|_{x=L}=\frac{C_{n-2}-4 C_{n-1}+3 C_{n}}{2 \Delta x}
\end{aligned}
$$

The exothermic reaction of $\mathrm{H}_{2}+\frac{1}{2} \mathrm{O}_{2} \rightarrow \mathrm{H}_{2} \mathrm{O}$ studied by Maymo and Smith (1966) was used with the kinetics of the reaction quoted as:

$$
r=0.327 P_{2}^{0.804} \exp \left(-\frac{5.230}{R T}\right)
$$

Table (1) shows the various parameters used in the simulations.

Table 1: Parameters Used in the Simulations

\begin{tabular}{|l|l|}
\hline $\begin{array}{l}\text { Bulk gas temperature } \\
=379-479 \mathrm{~K}\end{array}$ & $\begin{array}{l}\text { Network length }= \\
0.93 \mathrm{~cm}\end{array}$ \\
\hline $\begin{array}{l}\text { Effective diffusivity }= \\
1-10 \mathrm{~cm}^{2} / \mathrm{s}\end{array}$ & $\begin{array}{l}\text { Bulk gas } \\
\text { concentration }= \\
1.72 \mathrm{e}-6 \mathrm{~mol} / \mathrm{cm}^{3}\end{array}$ \\
\hline $\begin{array}{l}\text { Effective thermal } \\
\text { conductivity= 0.0051 } \\
\text { cal/s cm K }\end{array}$ & \\
\hline $\begin{array}{l}\text { Single sized pores }= \\
250 \mathrm{~A}^{0} \text { to } 2500 \mathrm{~A}^{0}\end{array}$ & \\
\hline $\begin{array}{l}\text { Random sized pore } \\
\text { network }=\text { pores } \\
\text { randomly distributed } \\
\text { between 200 and } \\
2500 \mathrm{~A}^{0}\end{array}$ \\
\hline
\end{tabular}

The resulting nonlinear algebraic equations were solved with Trust-Regions optimization method.
The individual pore effectiveness factor in the network is obtained from:

$$
\eta_{i}=\frac{-\pi r_{i}^{2} \bar{D}\left[\left(\frac{d C_{i}}{d x}\right)_{x=0}-\left(\frac{d C_{i}}{d x}\right)_{x=L}\right]}{2 \pi r_{i} l_{i} r\left(C_{s}, T_{s}\right)}
$$

Similarly the network effectiveness factor is calculated from:

$\eta=\frac{\sum_{i=1}^{n} V_{i} \eta_{i}}{V_{\text {total }}}$

Upon estimation of the exit concentrations from each pore and nodes and for a given network effectiveness factor the corresponding Thiele modulus for the catalyst pellet was estimated from the root of the equation:

$$
\eta=\frac{3 \phi \operatorname{coth}(3 \phi-1)}{3 \phi^{2}}
$$

This equation is a generalized expression for pores of any shape and extended to any form of rate expressions.

Sharrat and Mann (1987) obtained the tortuosity factors in their simulation studies of a first order reaction by:

$$
\frac{\phi_{\text {obs }}}{\phi_{\text {theor }}}=\frac{\left[L \sqrt{\frac{2 k_{s} \tau}{r D_{e} \varepsilon}}\right]_{o b s}}{\left[L \sqrt{\frac{2 k_{s} \tau}{r D_{e} \varepsilon}}\right]_{\text {theor }}}
$$

In which the theoretical Thiele modulus can be calculated from the kinetics of the reaction and consequently the observed tortuosity factor for the catalyst is obtained from:

$$
\tau=\left[\frac{\phi_{\text {obs }}}{\phi_{\text {theor }}}\right]^{2}
$$


The Jacobian matrix of the equations was solved analytically and this improved the iteration time remarkably. The solution routine required good initial estimates for a rapid convergence and hence first the nonreactive equations were solved and the exit concentrations were used as the initial estimates for the reactive simulations. Figure 2 shows a schematic of the Jacobian matrix that can be seen to be sparse and not diagonally dominant. Three densities of the network were used; 10x10, 20x20 and $30 \times 30$ and the simulations were repeated 10 times to obtain average effectiveness factors.

Two types of simulations were carried out. In the first type the effective diffusivity was kept constant and the bulk gas temperature was varied between 379$479 \mathrm{~K}$ and in the second type the bulk gas temperature was kept constant and the effective diffusivity was varied between 1$10 \mathrm{~cm}^{2} / \mathrm{s}$. These simulations were carried out for network consisting of single sized pores and also for network with randomly generated pores sizes.

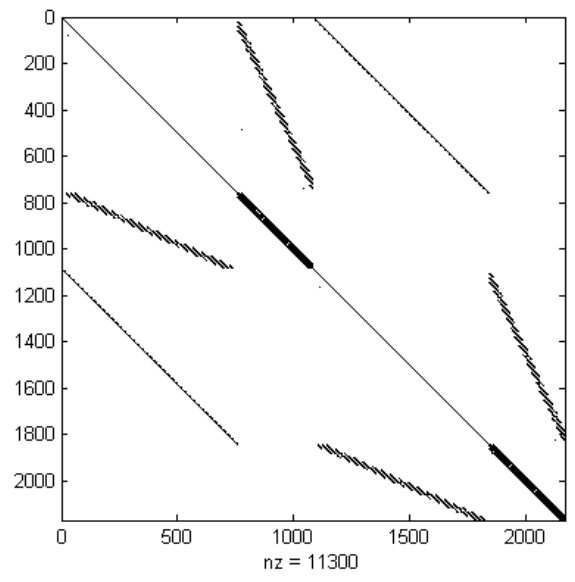

Figure 2: Structure of the Jacobian Matrix

Figure 3 shows the network effectiveness factors obtained. It can be seen that lower values have been obtained for the 10x10 network. Similar values were obtained for the 20x20 and 30x30 networks and therefore the rest of simulations were carried out on the $30 \times 30$ grid. It can also be seen from this figure that for the range of temperature and effective diffusivities used steady state multiplicity in the catalyst is not observed.

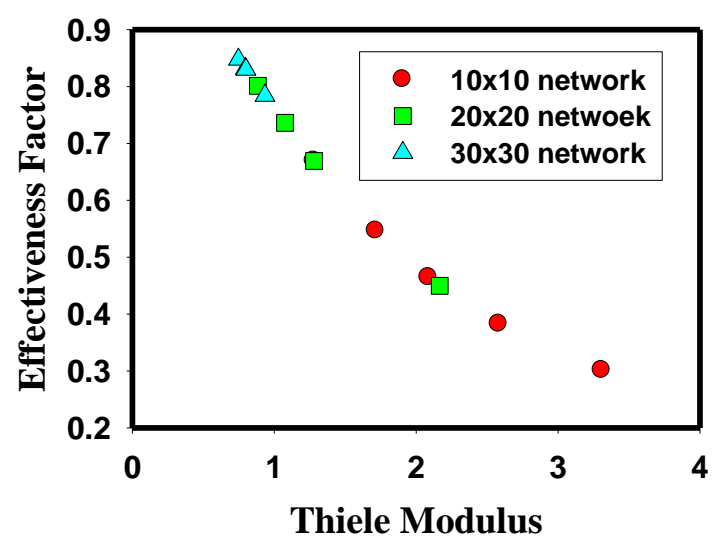

(a)

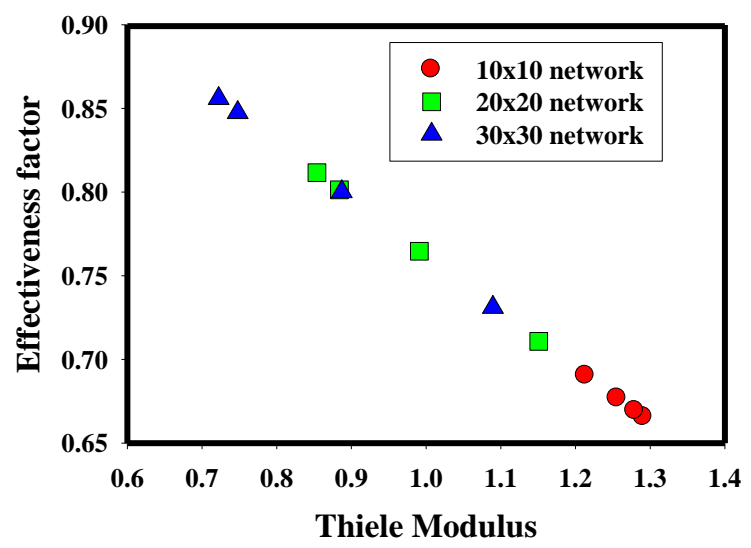

(b)

Figure 3: Variation of effectiveness factor with Thiele modulus for single sized pores of diameter $2500 \mathrm{~A}^{0}$ (a) bulk gas temperature of $390 \mathrm{~K}$ and effective diffusivity values from 1-10 $\mathrm{cm}^{2} / \mathrm{s}$ (b) Variation of effectiveness factor with Thiele Modulus effective diffusivity of 10 and bulk gas temperature from 379 to $479 \mathrm{~K}$

To study the effect of pore size on the tortuosity values, simulations were carried out for single sized pore networks as shown in Figure 4 and 5. Simulations in Figure 4 were carried out keeping the bulk gas temperatures constant while the effective diffusivity was varied. The 
tortuosity factor increases with increasing Thiele modulus for the network of pores of size $250 \mathrm{~A}^{0}$. At this pore size, $250 \mathrm{~A}^{0}$, the diffusion limitations are controlling and at higher pore sizes surface reaction limitations are controlling. The pore size of, $250 \mathrm{~A}^{0}$, is usually taken in the literature as the cut off size for the micro and macropores.

At the macropore size range at the low temperature of $379 \mathrm{~K}$ variation of diffusivity does not seem to affect the tortuosity values whereas at the temperature of $479 \mathrm{~K}$ the tortuosity factor is predicted to decrease with increasing diffusivity. Further simulation carried our, explained later in this article, will approve of these predictions.

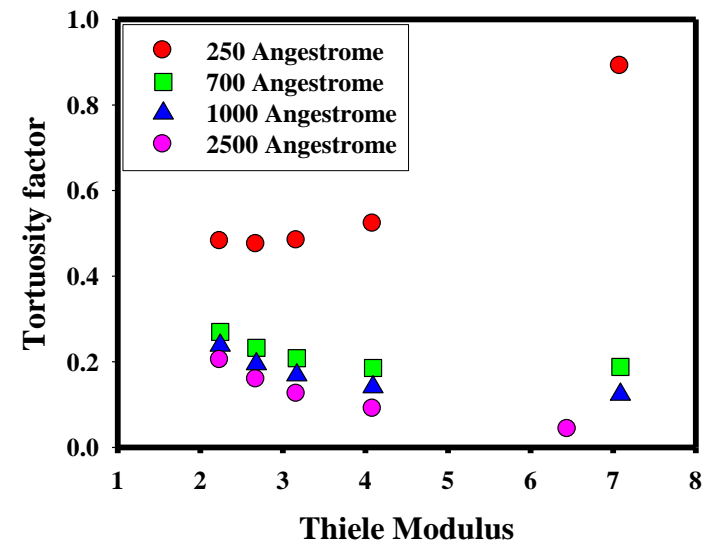

(a)

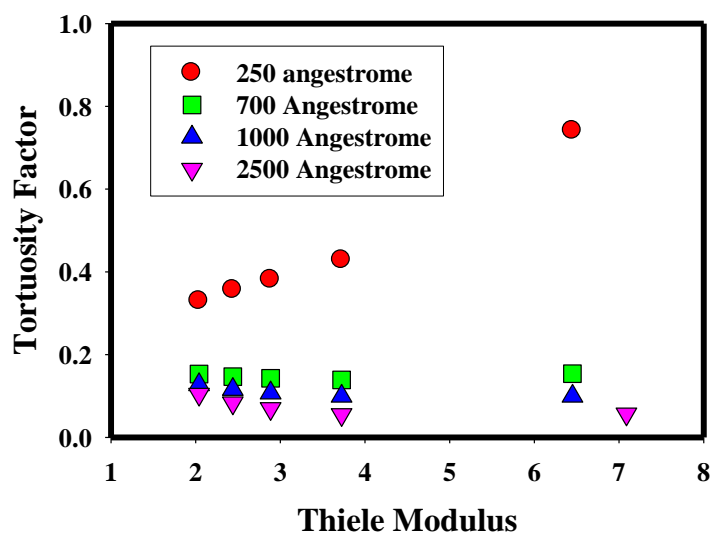

(b)

Figure 4: Variation of Tortuosity factor of the single sized pore networks with effective diffusivity $\left(1-10 \mathrm{~cm}^{2} / \mathrm{s}\right)$ at constant bulk gas temperature of (a) $479 \mathrm{~K}$ (b) temperature of 379 K

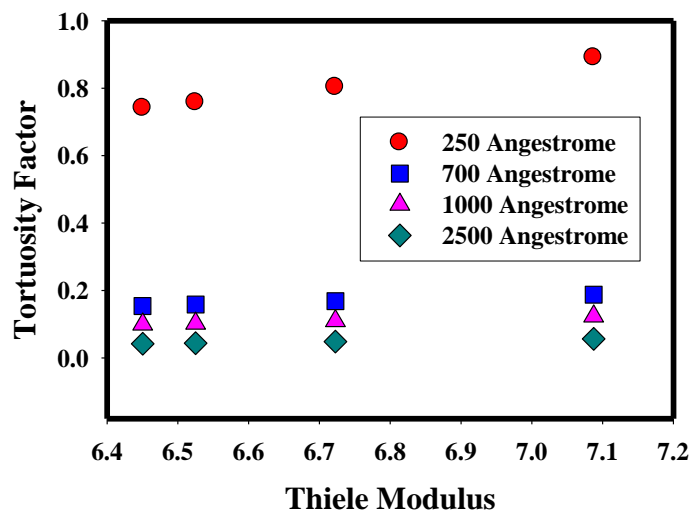

(a)

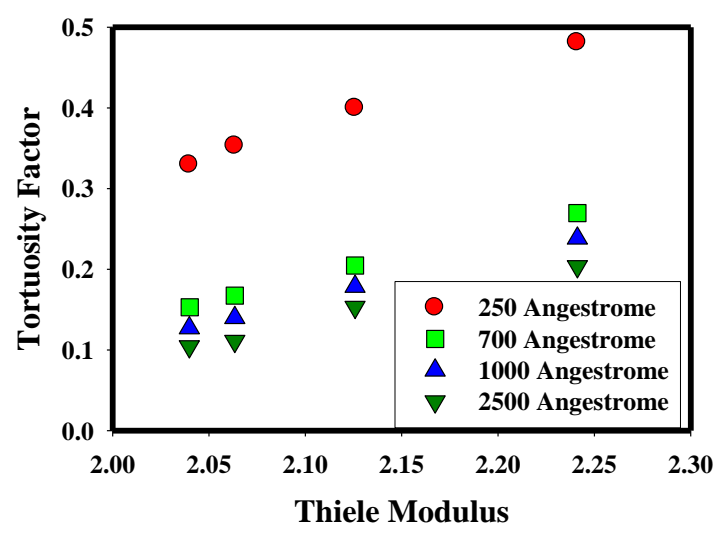

(b)

Figure 5: Variation of Tortuosity factor of the single sized pore networks with bulk gas temperature (379 to $478 \mathrm{~K})$ at constant diffusivity of (a) $1 \mathrm{~cm}^{2} / \mathrm{s} \mathrm{(b)} 10 \mathrm{~cm}^{2} / \mathrm{s}$

Figure 5 shows the simulation results at fixed diffusivity whilst varying the bulk gas temperature. It can be seen that at low diffusivity variation of temperature has insignificant effect on the tortuosity values whilst at the higher diffusivity of $10 \mathrm{~cm}^{2} / \mathrm{s}$ tortuosity values change with temperature. The $250 \quad \mathrm{~A}^{0}$ pore size network show significantly higher tortuosity values as obtained in Figure 4. 
Figures 6 and 7 show variation of tortuosity factor with diffusivity and temperature for the isothermal and nonisothermal pellets of pore size of $250 \mathrm{~A}^{0}$. Tortuosity factor is predicted to increase with decreasing diffusivity values as shown in Figure 6a and this follows from equation (1) that tortuosity varies inversely with effective diffusivity. The predicted tortuosity factors under isothermal conditions show less variation and also lower values in comparison with nonisothermal values.

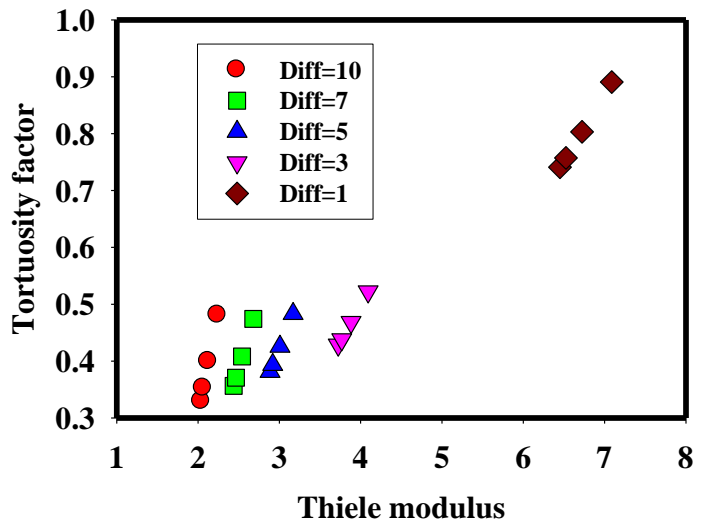

(a)

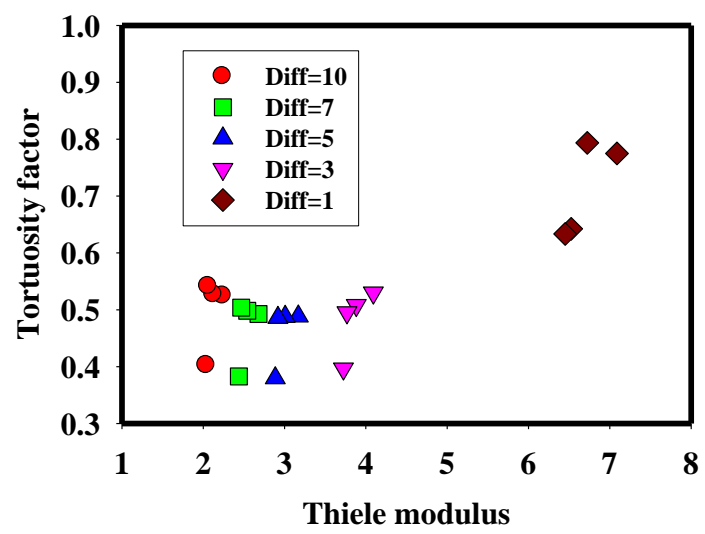

(b)

Figure 6: Variation of Tortuosity Factor with Diffusivity for the Network with Pores of Diameters of $250 \mathrm{~A}^{0}$ (Bulk Gas Temperature 379 to $479 \mathrm{~K}$ ) ;(a) Non-Isothermal Pellet (b) Isothermal Pellet

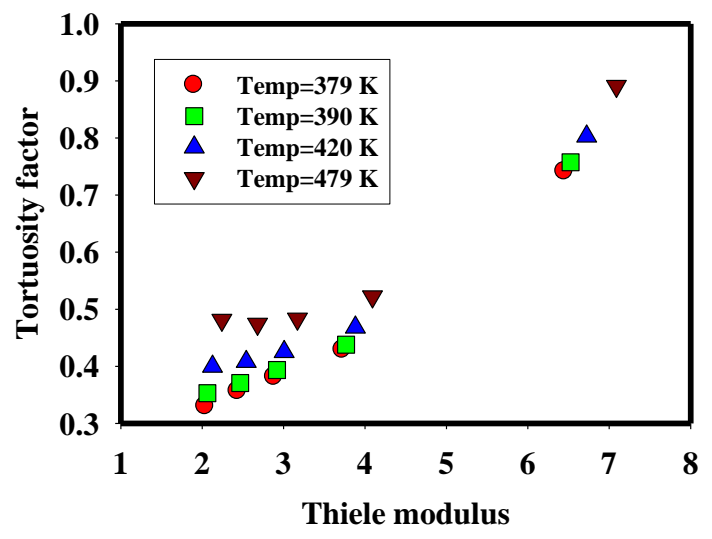

(a)

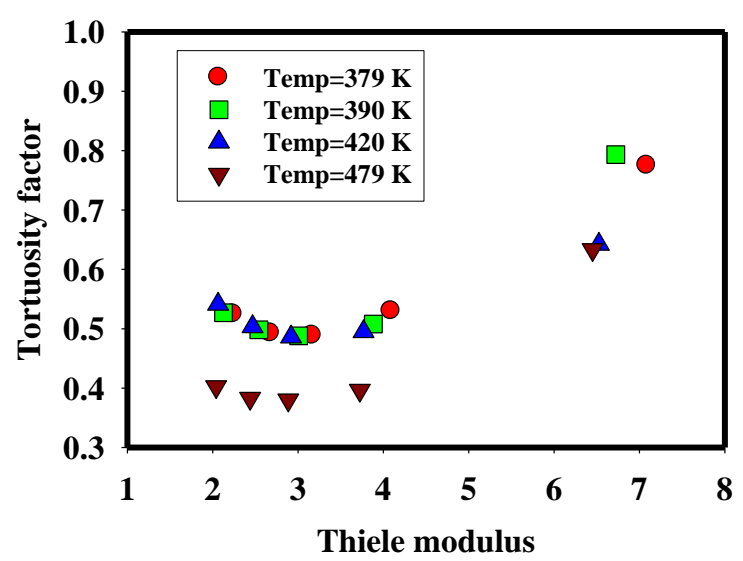

(b)

Figure 7: Variation of Tortuosity Factor with Bulk Gas Temperature for the Network with Pores of Diameter of $250 \mathrm{~A}^{0}$ (diffusivity 1 to 10 $\mathrm{cm}^{2} / \mathrm{s}$ ); (a) Non-isothermal pellet (b) Isothermal Pellet

As shown in Figure 7a increasing the bulk gas temperature increases the tortuosity factor estimated. At this pore size Knudsen diffusion dominates in the pores and at higher Thiele modulus values (diffusion limitations) the tortuosity values attain similar values. However, as shown in Figure $7 \mathrm{~b}$ the estimated tortuosities estimated under the isothermal pellet condition remain fairly unchanged with the changing bulk gas temperature. 


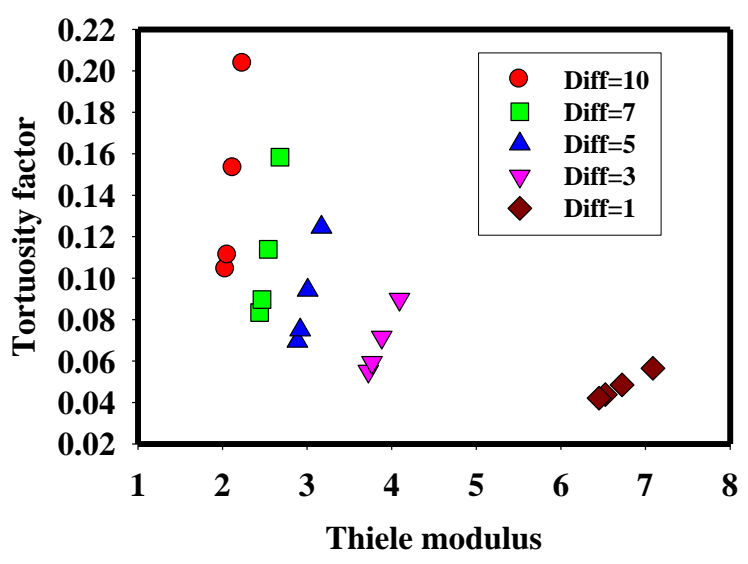

(a)

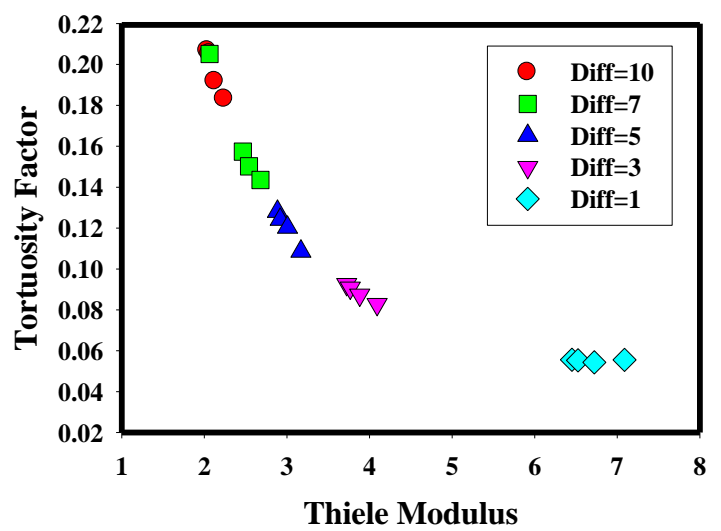

(b)

Figure 8: Variation of Tortuosity Factor with Diffusivity for the Network with Pores of Diameters of $2500 \mathrm{~A}^{0}$ (Bulk Gas Temperature 379 to $479 \mathrm{~K}$ ) ;(a) Non-Isothermal Pellet (b) Isothermal Pellet

Figures 8 and 9 show the simulation results for the isothermal and non-isothermal pellets for the network consisting of pores of the same size of $2500 \mathrm{~A}^{0}$. It is expected from equation (1) that tortuosity should increase with decreasing diffusivity but as shown in Figure $8 \mathrm{a}$ the contrary occurs and the highest tortuosities are obtained at the highest diffusivity of $10 \mathrm{~cm}^{2} / \mathrm{s}$. Additionally Figure 9a shows variation of tortuosity with bulk gas temperature but with a negative gradient in contrast to the network with pores of size $250 \mathrm{~A}^{0}$ as shown in Figure $7 \mathrm{a}$ where a positive gradient in obtained. At this range of pore sizes, $2500 \mathrm{~A}^{0}$, bulk diffusion in the pores dominates and reaction step becomes controlling.

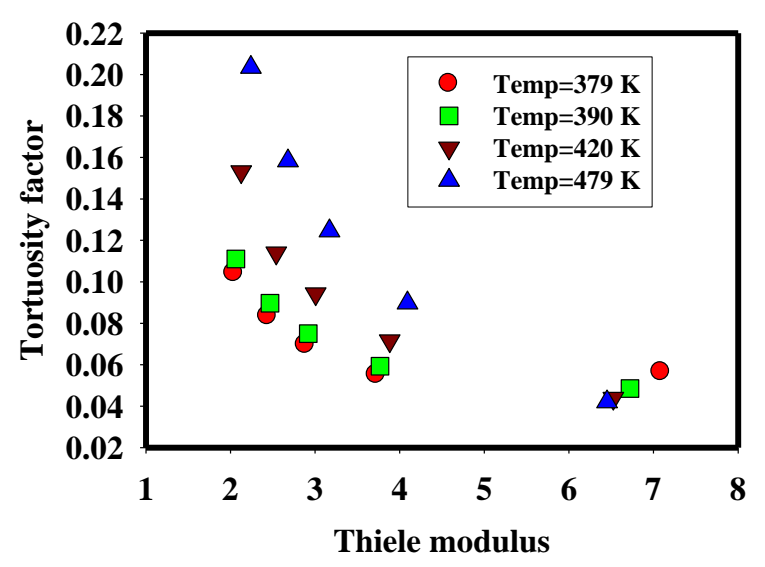

(a)

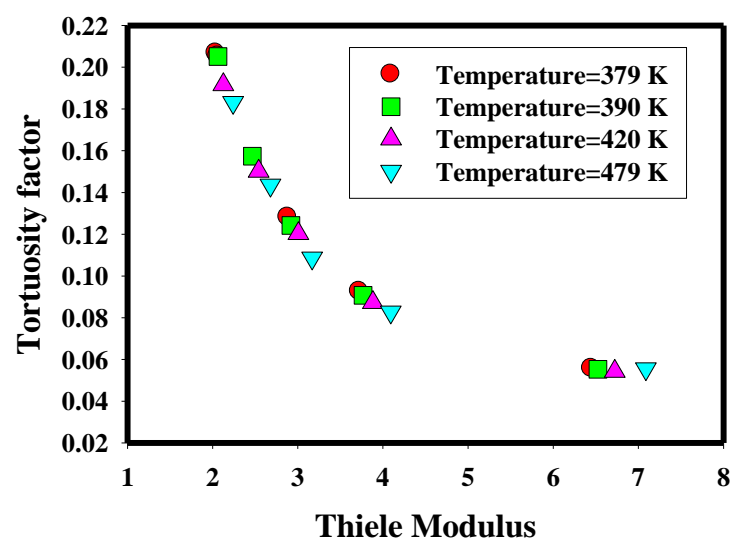

(b)

Figure 9: Variation of Tortuosity Factor with Bulk Gas Temperature for the Network with Pores of Diameter of $2500 \mathrm{~A}^{0}$ (diffusivity 1 to $10 \mathrm{~cm}^{2} / \mathrm{s}$ ); (a) Non-isothermal Pellet (b) Isothermal Pellet

Figure $8 \mathrm{~b}$ predicts a slight variation of tortuosity with diffusivity under the isothermal condition and Figure 9b predicts no variation of tortuosity with bulk gas temperature under the isothermal condition and similar to non-isothermal pellet the negative gradient is also obtained under the isothermal pellet conditions. 


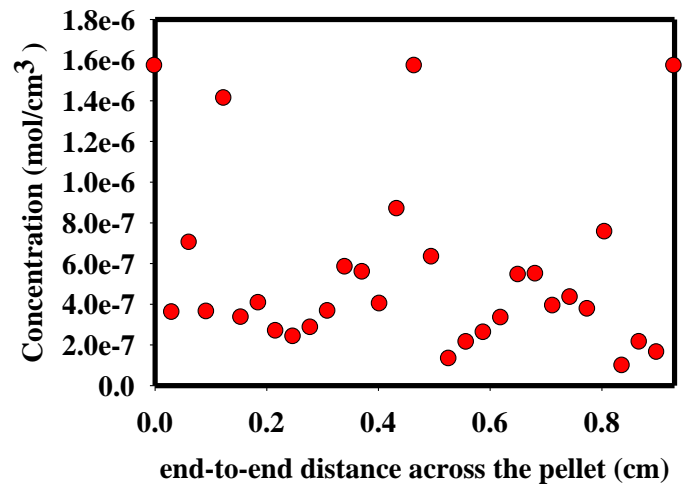

(a)

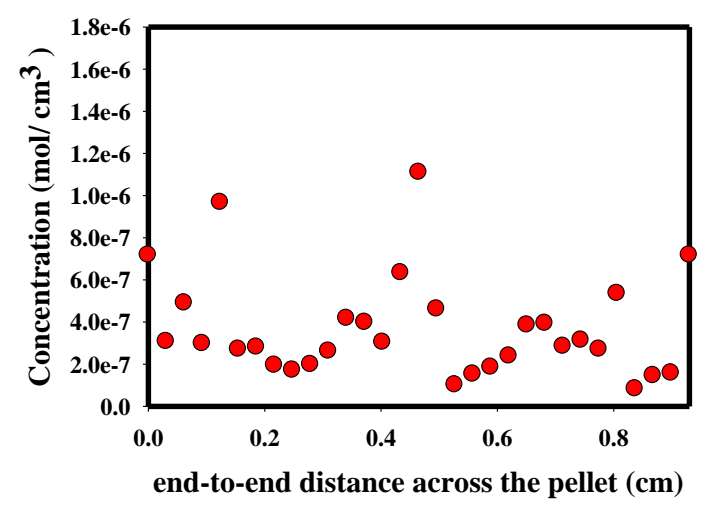

(b)

Figure 10: Concentration Profile Across the Central Part of Pellet at Diffusivity of 10 and Temperature of $479 \mathrm{~K}$ (a) Pores of size $2500 \mathrm{~A}^{0}$ (b) Pores of size $250 \mathrm{~A}^{0}$

Figure 10 and 11 show the concentration and temperature profiles for the central part of the pellet. Generally speaking, the network with pore sizes of $2500 \mathrm{~A}^{0}$ show higher concentrations and lower temperatures, indicating less reaction occurring, than the network with pore sizes of $250 \mathrm{~A}^{0}$. Due to higher rates of reactions in the network with small pores this network show hot spot temperatures of $700 \mathrm{~K}$.

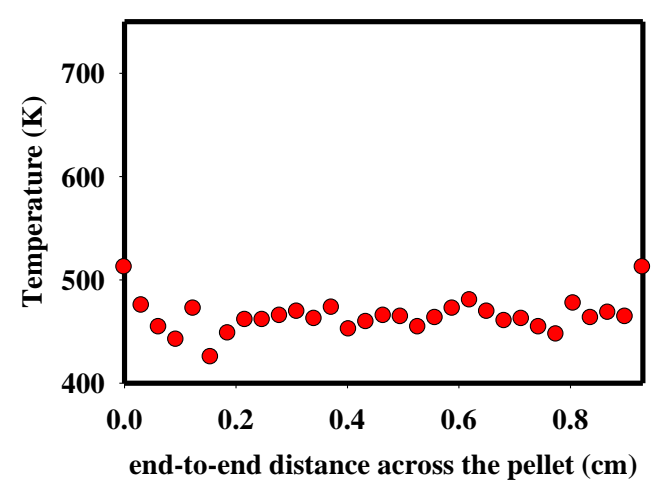

(a)

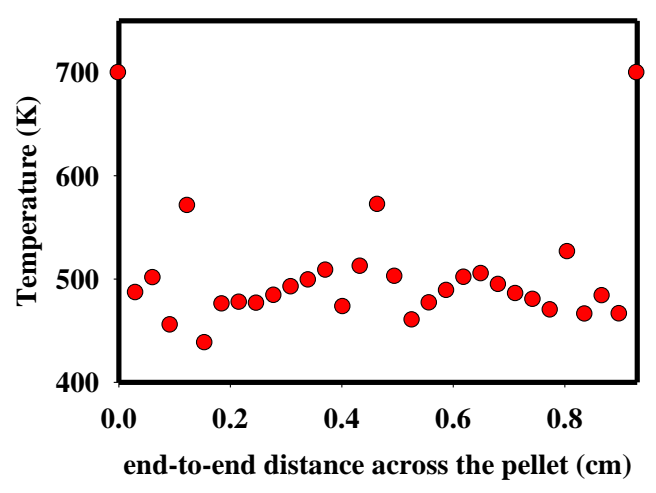

(b)

Figure 11: Temperature Profile across the Central Part of Pellet at Diffusivity of 10 and Temperature of $479 \mathrm{~K}$ (a) Pores of Size $2500 \mathrm{~A}^{0}$ (b) Pores of Size $250 \mathrm{~A}^{0}$

Further simulations were carried out with pores having random sizes between 200 to $2500 \mathrm{~A}^{0}$. The large pore sizes establish the bulk gas concentrations rapidly and hence they are important for the transport step and smaller pore sizes are more active in the reaction step. Therefore a combination of these pores should show higher overall activity. Close observation of Figure 12 and 13 reveals that similar trend to pore network of size $2500 \mathrm{~A}^{0}$ is obtained for these random networks. 


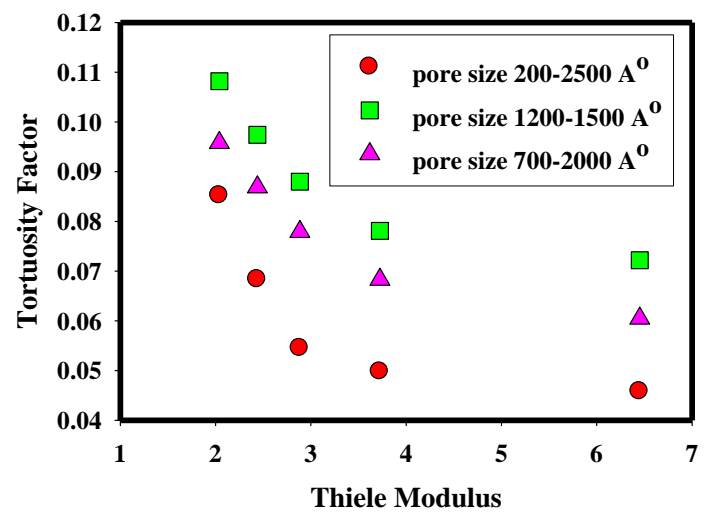

(a)

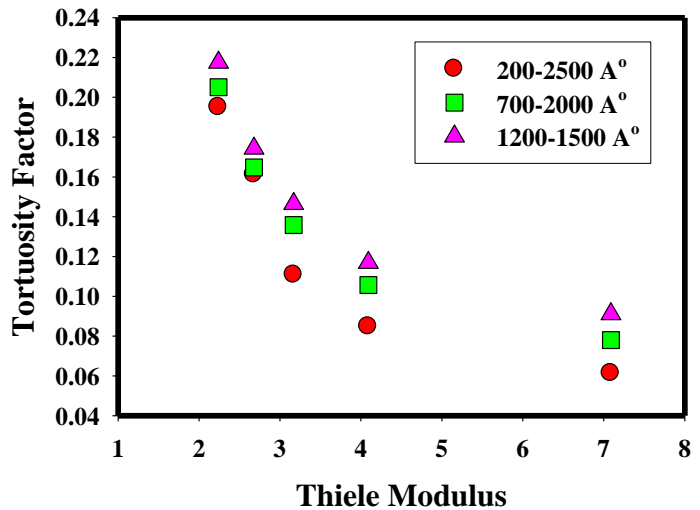

(b)

Figure 12: Random Sized Pores at Constant Temperature of (a) $379 \mathrm{~K}$, (b) $479 \mathrm{~K}$

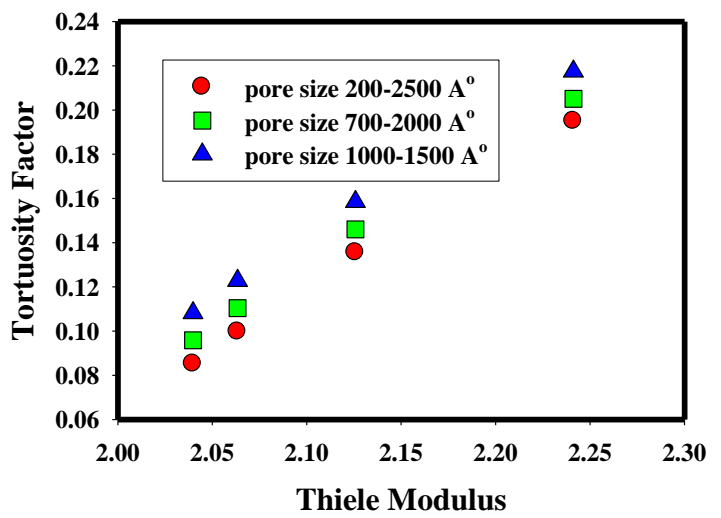

(a)

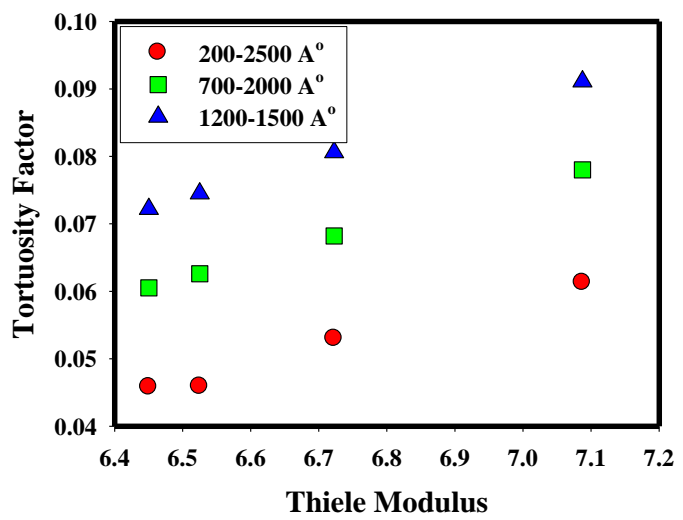

(b)

Figure 13: Random Sized Pores at Constant Diffusivity of (a) 10, (b) $1 \mathrm{~K}$

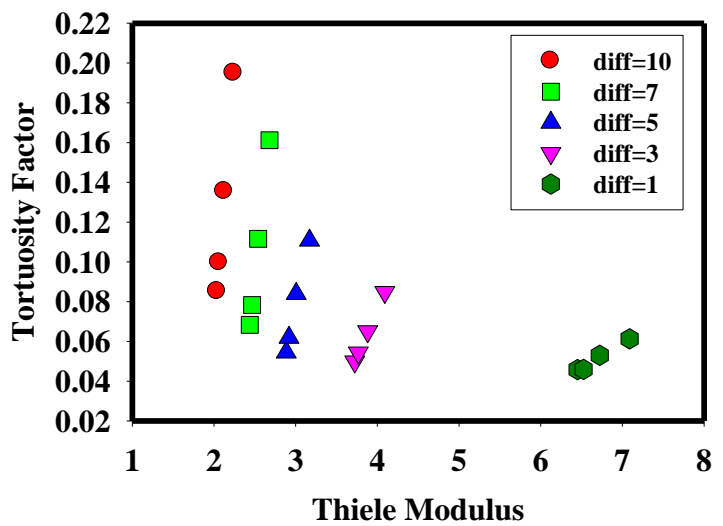

(a)

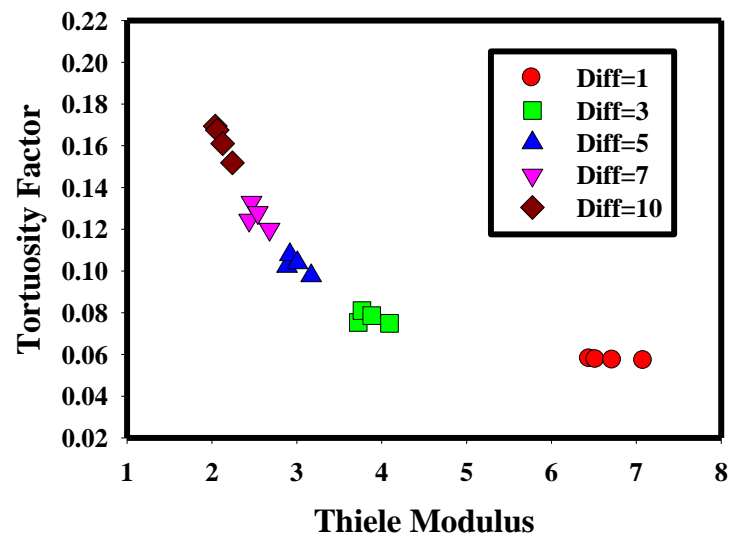

(b)

Figure 14: Variation of Tortuosity Factor with Diffusivity for the Network with Random Pore Sizes 200-2500 A ${ }^{\mathrm{o}}$ (Bulk Gas Temperature 379 to $479 \mathrm{~K}$ ) ;(a) Non-Isothermal Pellet (b) Isothermal Pellet 


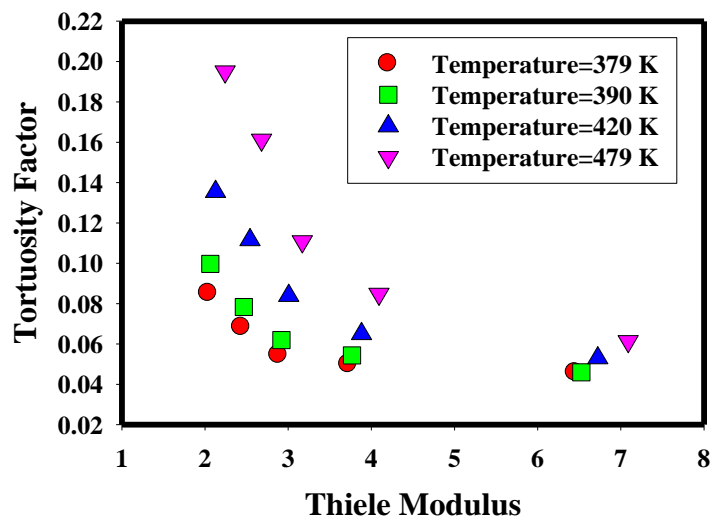

(a)

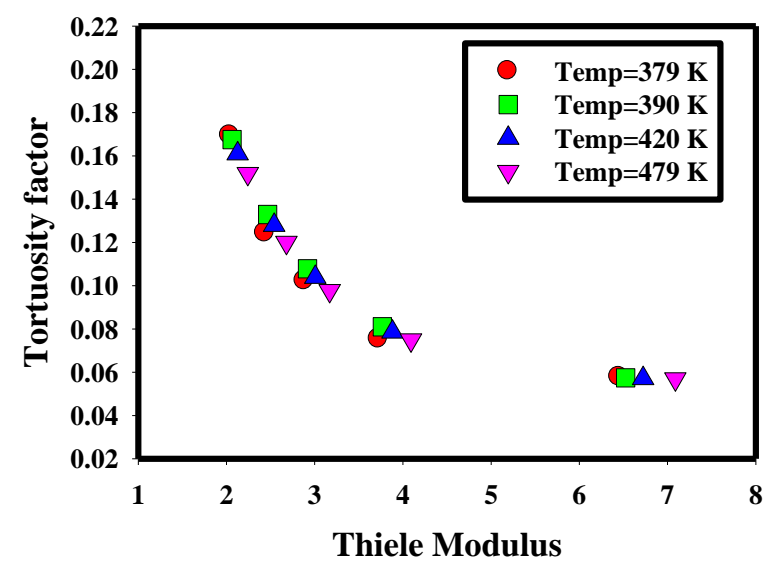

(b)

Figure 15: Variation of Tortuosity Factor with Bulk Gas Temperature for the Network with Random Pore Sizes 200-2500 A ${ }^{\circ}$ (Diffusivity 1 to $10 \mathrm{~cm}^{2} / \mathrm{s}$ ); (a) Non-Isothermal Pellet (b) Isothermal Pellet

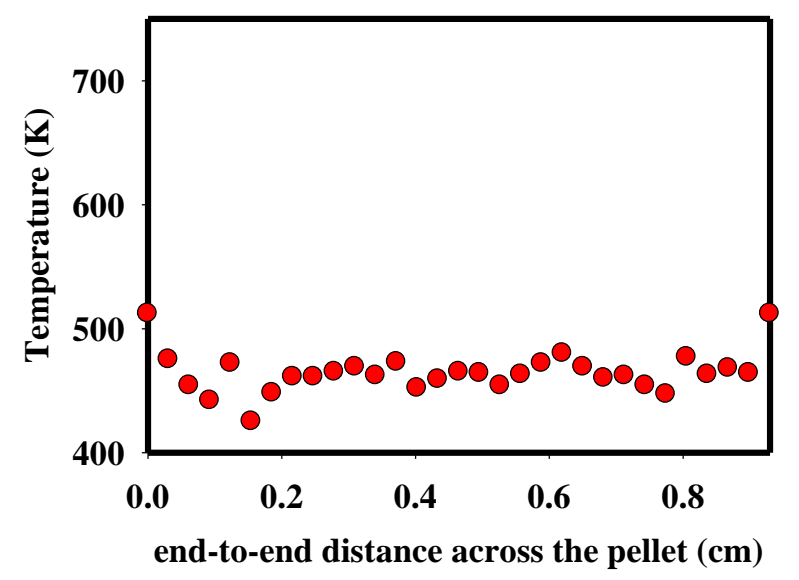

(a)

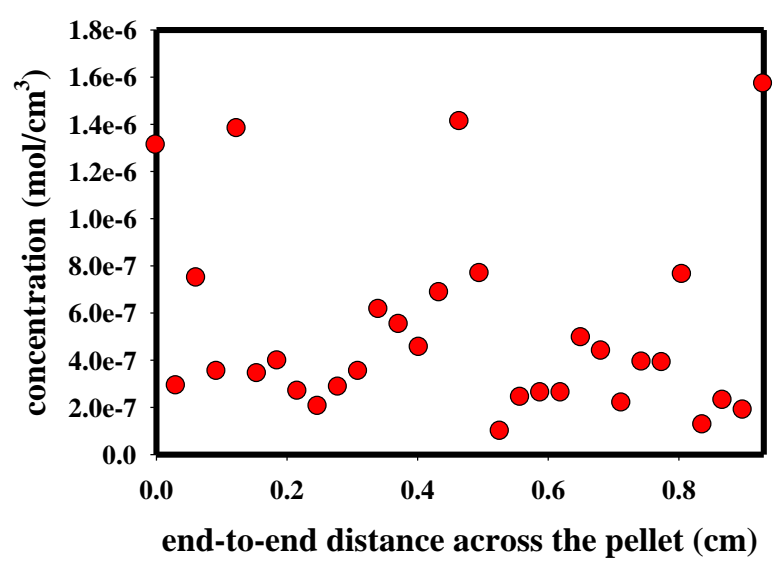

(b)

Figure 16: Temperature and Concentration Profile across the Central Part of Pellet at Diffusivity of 10 and Temperature of $479 \mathrm{~K}$ for the $200-2500$ A $^{\circ}$ Network

\section{CONCLUSION}

A two dimensional pore network model has been developed to study the effect of interparticle temperature gradient on the prediction of the tortuosity values. The simulations support the findings by Mann (1987) and Hollewand and Gladden (1992) that tortuosity factor is dependent on the Thiele modulus.

The size of pores has great impact upon the estimated tortuosity values. Large pore sized network, $2500 \mathrm{~A}^{0}$, show significantly lower tortuosities values and less variation of tortuosities with the bulk gas temperature. Additionally at constant bulk gas temperature this network shows tortuosity values decreasing with increasing Thiele modulus (or decreasing diffusivities). At constant diffusivities this network shows increasing tortuosity values with increasing bulk gas temperatures. Highest tortuosities are obtained at the highest diffusivities.

In the small pore sized network, 250 $\mathrm{A}^{0}$, higher tortuosity values are obtained and also significant variation of the estimated 
tortuosities with bulk gas temperature is obtained. Also, at constant bulk gas temperature this network shows tortuosity values increasing with increasing Thiele modulus (or decreasing diffusivities).

At constant diffusivities this network show increasing tortuosity values with increasing bulk gas temperatures. Highest tortuosities obtained at the lowest diffusivities.

The random sized network was expected to show trends between the small and the large pore sized networks but similar trends to the large single sized network were obtained. The decrease in the tortuosity values with increasing Thiele modulus has been attributed to the so-called "pore shielding" of the larger pores by smaller ones (see Mann [1987] and Gladden [1992]). However, it has been shown in this work that for the large single sized pore network, eg $2500 \mathrm{~A}^{0}$, the tortuosity values also decrease with increasing Thiele modulus. In this work this behaviour has been attributed to reaction term being the limiting step for this network. However, the mechanism of effect of the Thiele modulus on the tortuosity values is not known since tortuosity is only a parameter of the geometry of the catalyst.

For the $250 \mathrm{~A}^{0}$ single sized network assumption of isothermal pellet may be justified since there is no significant difference between the simulations carried out under isothermal and non-isothermal catalyst assumption. However, the $2500 \mathrm{~A}^{0}$ single sized network reveals that at constant bulk gas temperature variation of diffusivity does not affect the tortuosity values for the isothermal catalyst whereas for the nonisothermal catalyst tortuosity values decreases with decreasing diffusivities. At fixed diffusivities the tortuosity values increases with increasing bulk gas temperature and the highest tortuosity is obtained for the highest diffusivities whereas for the isothermal catalysts there is no significant variation of the tortuosity values.

The random pore sized networks show similar variation of tortuosity values to the large pore sized networks, under both isothermal and non-isothermal assumptions, and therefore isothermal catalyst assumptions has been shown to produce different tortuosity values from nonisothermal catalyst assumption for this exothermic reaction.

\section{Nomenclature}

C concentration

$C_{b} \quad$ bulk gas concentration

$C_{s} \quad$ outer node concentration

$D(r) \quad$ bulk gas diffusivity

$D_{e} \quad$ effective diffusivity

$J_{i} \quad$ flux

$L \quad$ pellet length

$K_{g} \quad$ mass transfer coefficient

$r_{p} \quad$ pore radius

$T$ temperature

$T_{b} \quad$ bulk gas temperature

$T_{s} \quad$ surface node temperarture

$V_{\text {total }}$ total pore volume

$V_{i} \quad$ individual pore volume

$X \quad$ distance into the pellet

$\tau \quad$ Tortuosity factor

$\rho \quad$ gas density

$\Delta H \quad$ heat of reaction

$\lambda_{e} \quad$ Effective thermal conductivity

$\eta \quad$ Effectiveness factor

$\phi \quad$ Thiele modulus

\section{REFERENCES}

[1] Thiele, E.W., Ind.Eng.Chem. 31, 916920, (1939)

[2] Petersen, E.E, A.I.Ch.E.J, 3,443-448, (1957) 
[3] Fatt, I, Petrol.Trans.AIME, 207, 144159, (1956)

[4] Beckman, J.W., Froment, G.F., Ind.Eng.Chem.Fund. 18, 245-256, (1979)

[5] Sharrat, P.N, Mann, R, Chem.Eng.Sci. 42, No.7, 1565-1576, (1987)

[6] Hollewand, M.P, Gladden, L.F, Chem.Eng.Sci, 47, 1761-1770, (1992)

[7] Rieckmann, C, Kiel, F.J, Chem.Eng.Sci, 54, 3485-3493, (1999)

[8] Satterfield, C.N, Cadle, P.J, Ind.Eng.Chem.Process Des.Dev, 7, 256-260, (1968)

[9] Otani, Smith, J.M, J.Catal, 5, 332-347, (1966)

[10] M.Sahimi, G.R.Gavalas, T.T.Tsotsis, Chem.Eng.Sci, 45, 1443-1502, (1990)

[11] J.A. Mayamo, J.M.Smith, AICheJ, 12, 845, (1966) 